\title{
Impact of increased erythropoietin receptor expression and elevated serum erythropoietin levels on clinicopathological features and prognosis in renal cell carcinoma
}

\author{
KEIICHI ITO, HIDEHIKO YOSHII, TAKAKO ASANO, AKIO HORIGUCHI, MAKOTO SUMITOMO, \\ MASAMICHI HAYAKAWA and TOMOHIKO ASANO
}

Department of Urology, National Defense Medical College, Tokorozawa, Saitama 359-8513, Japan

Received January 23, 2012; Accepted February 27, 2012

DOI: $10.3892 / \mathrm{etm} .2012 .513$

\begin{abstract}
Erythropoietin (EPO) expression and EPO receptor (EpoR) expression have been demonstrated in various malignant tumors. EPO-EpoR signaling can activate several downstream signal transduction pathways that enhance tumor aggressiveness. The present study was undertaken to evaluate the impact of overexpression of EpoR and elevated serum EPO (sEPO) levels on the clinicopathological features and prognosis of patients with renal cell carcinoma (RCC). EpoR expression was evaluated immunohistochemically in 56 patients. Tumors with a staining intensity greater than that of surrounding proximal tubules were defined as tumors with high EpoR expression. The association between EpoR expression levels and various clinicopathological factors was analyzed. sEPO levels were determined in 138 patients and its correlation to clinicopathological factors was also analyzed, and EpoR expression was determined in surgical specimens removed from 47 of those 138 patients. Patients with high EpoR expression and patients with sEPO elevation had clinicopathological features less favorable than those of other patients. Tumors demonstrating high EpoR expression had a significantly higher number of Ki-67-positive cells compared to those with low EpoR expression. Tumor assemblies in microvessels demonstrated high EpoR expression. Patients whose tumors demonstrated high EpoR expression and those with sEPO elevation had a significantly lower survival rate compared to other patients, and patients with both high EpoR expression and sEPO elevation had an extremely poor prognosis. Microvascular invasion was an independent factor associated with sEPO elevation, suggesting that EPO-EpoR signaling might be important in RCC metastasis. EPO-EpoR signaling may be involved in tumor growth and progression in
\end{abstract}

Correspondence to: Dr Keiichi Ito, Department of Urology, National Defense Medical College, 3-2 Namiki, Tokorozawa, Saitama 359-8513, Japan

E-mail: itok@ndmc.ac.jp

Key words: renal cell carcinoma, erythropoietin, erythropoietin receptor, prognosis, microvascular invasion
RCC and the combination of EpoR expression and sEPO levels may effectively predict clinical outcome.

\section{Introduction}

Although polycythemia does not occur frequently in renal cell carcinoma (RCC) patients, erythropoietin (EPO) has been reported to be produced by tumor cells in the kidneys of polycythemic RCC patients (1), and EPO expression in RCC cells has also been demonstrated in vitro (2). Various types of human cancer cells have recently been found to express EPO, and EPO signaling has been suggested to be important in proliferation, invasion and angiogenesis (3). Although RCC rarely produces enough EPO to cause polycythemia, it is possible that EPO produced by RCC cells stimulates their aggressive behavior by acting on an autocrine and paracrine loop. Hypoxia-inducible factor (HIF) is often upregulated in RCC cells and EPO is one of the downstream gene products of HIF. sEPO levels are elevated in certain patients with RCC. This elevation is correlated with tumor stage and histopathological grade, and provides prognostic information (4). In a recent study, the positive cytoplasmic expression of EPO in surgical specimens was revealed to be associated with a poor prognosis for RCC patients (5).

RCC cells may become more sensitive to extracellular EPO if its receptor (EpoR) expression is upregulated. Increased EpoR expression in surgical specimens is associated with worse pathology and poorer prognosis in lung cancer (6) and endometrial carcinoma (7). EPO-EpoR signaling activates the JAK-STAT pathway in head and neck squamous cell carcinomas (8), and EPO activates the ERK/MAPK pathway in MCF-7 breast cancer cells (9) and stimulates RAF-1 tyrosine phosphorylation in erythroleukemic cells (10). Coexpression of EPO and EpoR was detected by RT-PCR in $92.6 \%$ of the surgical specimens removed from clear cell RCC patients regardless of VHL mutation status (11), and marked EpoR expression has been reported to indicate poor prognosis for RCC patients (12). EPO-EpoR signaling also stimulates proliferation in RCC cells in vitro (2), therefore it appears that EPO-EpoR signaling may be imporant in RCC biology.

The present study was undertaken to evaluate the impact of increased EpoR expression by immunohistochemistry on the 
clinicopathological features and prognosis of RCC patients. Also to evaluate the impact of elevated sEPO levels on clinicopathological features and prognosis, and determine whether the combination of EpoR expression level and sEPO level can improve the accuracy with which clinical outcome can be predicted. In addition we also aimed to determine factors influencing the level of sEPO.

\section{Patients and methods}

Patients. We evaluated patients who underwent surgical removal of RCC at the National Defense Medical College, Tokorozawa, Saitama, Japan, between 1994 and 2006, in order to determine clinicopathological factors from clinical records and pathological reports. Paraffin-embedded sections were prepared from surgical specimens removed from 54 patients who underwent radical nephrectomy $(\mathrm{RN})$ and 2 who underwent partial nephrectomy (PN). Follow-up intervals for the 56 patients ranged from 1 to 162 months (median, 62.3 months). Paraffin-embedded sections of 5 metastatic lesions ( 2 in lymph nodes, 1 in the adrenal gland, 1 subcutaneous and 1 in the pancreas) from 5 different patients were also prepared. SEPO levels were available for 138 patients (121 patients underwent RN and 17 underwent PN), and EpoR expression was determined in surgical specimens removed from 47 of the 138 patients. All serum samples were obtained within a week prior to nephrectomy. Follow-up intervals for the 138 patients ranged from 1 to 129 months (median, 22.3 months). All patients were postoperatively evaluated for local recurrence and metastasis every 3 to 6 months for the first 5 years and every 6 to 12 months after. Follow-up examinations consisted of physical examination, chest radiography, abdominal and chest CT, blood tests and, if indicated, radionuclide bone scanning. The pathological stage was determined according to the 2002 TNM classification system, and a 3-graded system was used for nucleolar grading (13). This study was approved by the institutional review board of the National Defense Medical College, Tokorozawa, Saitama, Japan.

Immunohistochemicalanalysisfor EpoR inparaffin-embedded tissues. The clinicopathological factors of the 56 patients whose surgical specimens were evaluated in this study are listed in Table I. The patients included 40 males and 16 females between 36 and 78 years of age (median, 61). The median follow-up interval was 62 months (range, 1 to 162). Twenty patients underwent right nephrectomy and 36 patients underwent left nephrectomy. The size of the primary tumor was $6.7 \pm 4.1 \mathrm{~cm}$ (range, 1.3-20; median 6.3). The predominant histological type was clear cell type in 54 tumors and papillary type in 2 . The paraffin-embedded sections contained both tumor and surrounding kidney tissue. Immunohistochemistry was performed as previously described (14). Briefly, paraffin-embedded sections were deparaffinized. Slides were placed in Dako Target Retrieval Solution (Dako Corp., Carpinteria, CA, USA) and heated for antigen retrieval. Endogenous peroxidase activity was quenched with Dako Peroxidase Blocking Reagent (Dako Corp.). Sections were incubated in $10 \%$ normal goat serum in phosphate-buffered saline and subsequently incubated overnight with rabbit polyclonal anti-EpoR antibody (C-20; Santa Cruz Biotechnology,
Santa Cruz, CA, USA). They were then stained using a Simple Stain Max PO kit (Nichirei Corp., Tokyo, Japan). Reaction products were visualized by immersing the slides in diaminobenzidine tetrahydrochloride. The sections were counterstained with hematoxylin. Tubular epithelial cells, in which EpoR is known to be abundant (15), served as a positive internal control. Samples incubated without primary antibody were also stained using the same method and were used for baseline staining. Immunostaining results in all tumor sections were evaluated by 2 individuals (K.I. and T.A.) blinded to all clinical and pathological variables. The staining intensity of each tumor was compared with that of surrounding proximal tubules. Tumors with a staining intensity greater than that of the surrounding proximal tubules (level 2) were defined as tumors with high EpoR expression (level 3). Those with a staining intensity equal to (level 2) or less than (level 1) that of proximal tubules were tumors with low EpoR expression. Staining level was determined by reviewing the entire slide at x200 magnification and the dominant level of EpoR expression in each tumor was determined. The sections from all 56 patients were also immunostained for Ki-67 and CD34. The primary antibody for Ki-67 (mouse monoclonal; Zymed Laboratories Inc., South San Francisco, CA, USA) was used at an appropriate dilution. Ten high-power fields (HPFs) in each slide were counted by two independent investigators whose results were averaged. The primary antibody for CD34 (monoclonal; Santa Cruz Biotechnology) was used at a dilution of 1:100, and CD34-positive neovessels in $10 \mathrm{HPFs}$ were counted as previously described (16).

Measurement of serum EPO levels. EPO levels in duplicate 50-ml serum samples were measured using an enzyme immunoassay kit (Diagnostic Products Corporation, Los Angeles, CA, USA) according to the manufacturer's instructions.

Statistical analysis. Results are presented as the mean \pm standard deviation. Variables of different groups were compared using the Mann-Whitney $\mathrm{U}$ test. The independence of fit of the categorical data was analyzed by the Chi-square test. The correlation between variables was analyzed using Spearman's rank correlation coefficients. Survival curves were constructed using the Kaplan-Meier method, and the differences between them were assessed using the log-rank test. Cox's proportional hazard regression model was used for univariate and multivariate analyses. Logistic regression analysis was used to identify independent factors influencing sEPO elevation. $\mathrm{P}<0.05$ was considered to indicate a statistically significant difference.

\section{Results}

Expression of EpoR in RCC and surrounding tubular epithelium. Normal renal tubular epithelium clearly expressed EpoR, mainly in the cytoplasm (Fig. 1A); this localization of EpoR is consistent with previous studies (12). EpoR expression in RCC cells was also observed in the cytoplasm. The expression intensity was level 1 in 21 tumors (Fig. 1B), level 2 in 20 tumors and level 3 in 15 tumors (Fig. 1C). RCC cell assemblies in the microvessels surrounding the primary tumors frequently demonstrated high EpoR expression (Fig. 1D). 
Table I. Comparison of the clinicopathological factors between patients with low and high EpoR expression.

\begin{tabular}{lccc}
\hline & $\begin{array}{c}\text { Low EpoR } \\
(\mathrm{n}=41)\end{array}$ & $\begin{array}{c}\text { High EpoR } \\
(\mathrm{n}=15)\end{array}$ & P-value \\
\hline Male/female & $31 / 10$ & $9 / 6$ & $0.2522^{\mathrm{a}}$ \\
Age (years) & $59.5 \pm 11.9$ & $60.0 \pm 8.5$ & $0.9999^{\mathrm{b}}$ \\
Side (right/left) & $14 / 27$ & $6 / 9$ & $0.6856^{\mathrm{a}}$ \\
Size $(\mathrm{cm})$ & $5.5 \pm 3.1$ & $9.9 \pm 4.7$ & $0.0012^{\mathrm{b}}$ \\
$\mathrm{pT} 1$ or $2 / 3$ or 4 & $30 / 11$ & $6 / 9$ & $0.0222^{\mathrm{a}}$ \\
$\mathrm{N}^{+}$ & 2 & 3 & $0.0789^{\mathrm{a}}$ \\
$\mathrm{M}^{+}$ & 5 & 6 & $0.0204^{\mathrm{a}}$ \\
$\mathrm{Histological}^{+}$grade $3^{+}$ & 7 & 9 & $0.0016^{\mathrm{a}}$ \\
$\mathrm{MVI}^{+}$ & 14 & 12 & $0.0023^{\mathrm{a}}$ \\
CRP $(\mathrm{mg} / \mathrm{dl})$ & $1.6 \pm 2.9$ & $4.7 \pm 7.0$ & $0.0073^{\mathrm{b}}$ \\
\hline
\end{tabular}

${ }^{a}$ Analyzed by the Chi-square test, ${ }^{b}$ analyzed by the Mann-Whitney U test. EpoR, erythropoietin receptor; pT, pathological T stage; $\mathrm{N}^{+}$, lymph node metastasis; $\mathrm{M}^{+}$, distant metastasis; MVI, microvascular invasion; CRP, C-reactive protein.

All five RCC metastases demonstrated high EpoR expression (level 3) (Fig. 1E). The RCC cell assemblies in the microvessels surrounding the metastatic lesions demonstrated higher EpoR expression than the metastatic lesions (Fig. 1F).

Comparison of clinicopathological factors and survival between patients with low and high EpoR expression. We compared the clinicopathological factors of 41 patients whose tumors demonstrated low EpoR expression (levels 1 and 2) and 15 patients whose tumors demonstrated high EpoR expression (level 3) (Table I). The patients with high EpoR expression had tumors with a significantly higher pathological T (pT) stage, size and histological grade than the patients whose tumors had low EpoR expression, and those patients also had higher C-reactive protein (CRP) levels and higher percentages of metastatic disease and microvascular invasion (MVI). Patients whose tumors demonstrated high EpoR expression had a cause-specific survival (CSS) rate significantly lower than patients whose tumors demonstrated low EpoR expression (Fig. 2A). In N0M0 patients $(n=41)$, however, disease-free survival (DFS) did not differ significantly between those with low EpoR expression and those with high EpoR expression (Fig. 2B).

Association between EpoR expression level and proliferation. The proliferative status of the cells in each of the excised tumors was evaluated by Ki-67 immunostaining (Figs. 3A-C). The number of Ki-67-positive RCC cells per HPF was $7.7 \pm 1.0$ in level-1, 16.4 \pm 2.8 in level-2 and 32.6 \pm 5.3 in level-3 tumors. Tumors with higher EpoR expression levels had significantly larger numbers of Ki-67-positive cells, suggesting that EpoR expression is associated with the proliferation of RCC cells.

Association between EpoR expression level and angiogenesis. Level of angiogenesis was evaluated by CD34 immunostaining. The number of CD34-positive neovessels per HPF was 22.1 \pm 2.5 in level-1, 16.6 \pm 2.6 in level-2 and 19.2 \pm 3.5 in level-3 tumors. RCC specimens with low EpoR expression frequently had a large number of neovessels (Fig. 3D), and certain specimens

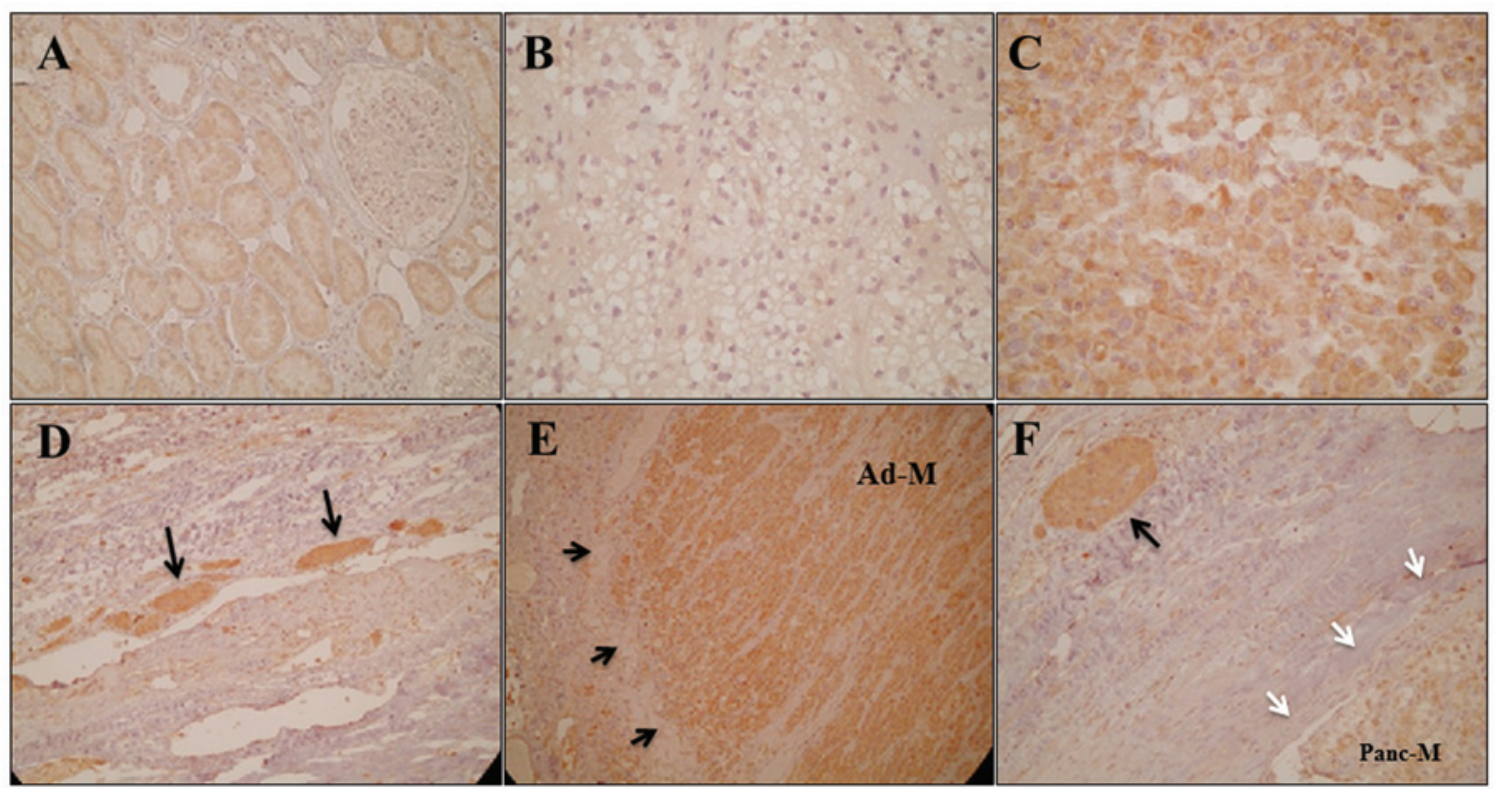

Figure 1. Immunolocalization of EpoR in tubular epithelial cells, primary lesions of RCC and metastatic lesions. (A) Normal renal tubular epithelium (reduced from x200) demonstrated cytoplasmic expression of EpoR; this level of expression was defined as level 2. The level of expression in a tumor demonstrating (B) lower EpoR expression than surrounding tubular cells was defined as level 1 (reduced from x200), and (C) higher EpoR expression than surrounding tubular cells was defined as level 3 (reduced from x200). (D) RCC cell assemblies (black arrows) in microvessels surrounding primary tumors frequently demonstrated high EpoR expression (reduced from x400). (E) Adrenal metastasis (Ad-M) demonstrated high EpoR expression (reduced from x100). Black arrows point to the margin of the metastasis. (F) RCC cell assembly (black arrow) in microvessels surrounding a pancreatic metastasis (reduced from $\mathrm{x} 400$ ). The assembly demonstrates higher EpoR expression than the metastasis. White arrows point to the margin of the pancreatic metastasis (Panc-M). EpoR, erythropoietin receptor; RCC, renal cell carcinoma. 
A

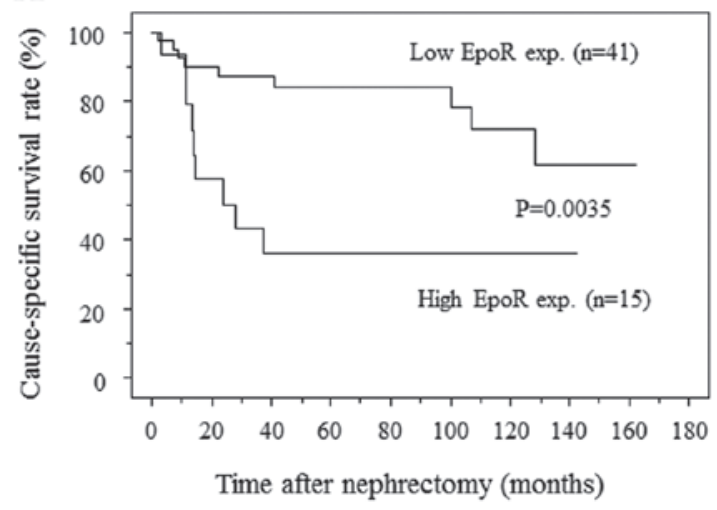

C

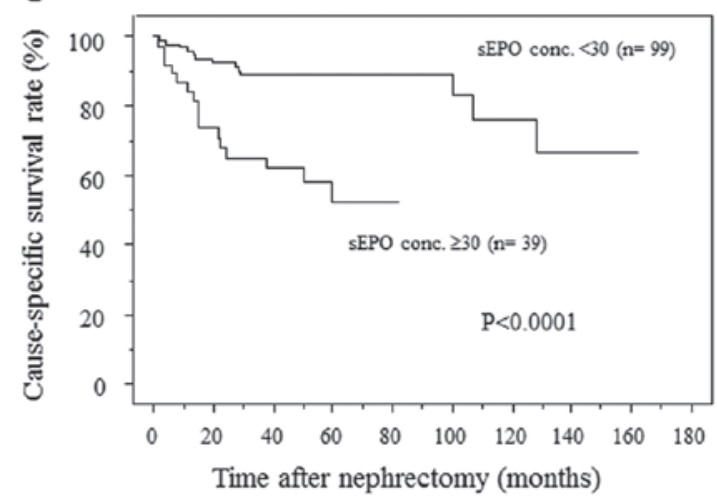

B

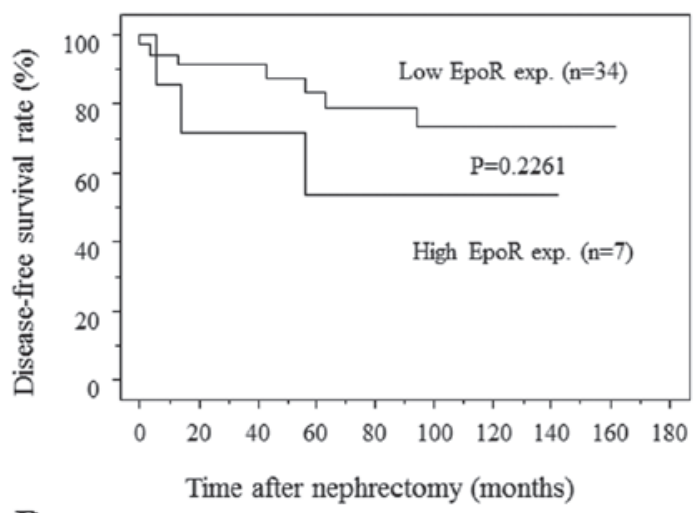

D

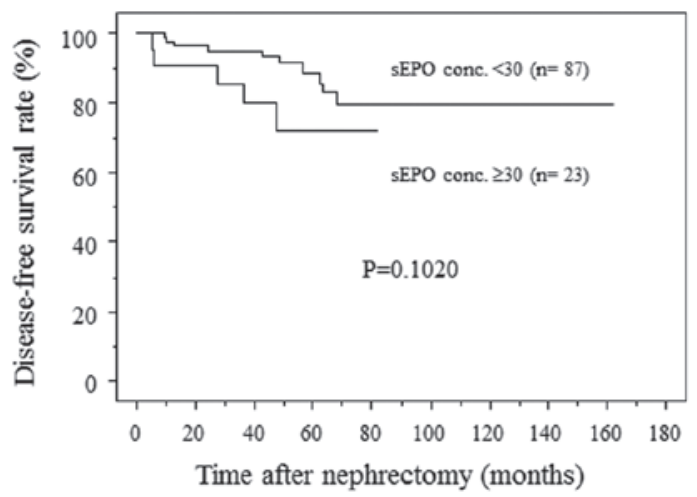

Figure 2. (A) RCC patients with tumors demonstrating high EpoR expression had significantly lower survival rates than those with tumor demonstrating low EpoR expression. The 1-, 3- and 5-year survival rates were 90, 87.3 and 84.2\%, respectively, for patients with tumors demonstrating low EpoR expression and 79, 43.1 and $35.9 \%$, respectively, for patients with tumors demonstrating high EpoR expression. (B) In N0M0 patients, however, DFS did not differ significantly between patients with tumors demonstrating high EpoR expression than those with low EpoR expression. (C) Patients with high sEPO levels had significantly lower rates of CSS than patients with low sEPO levels. The 1-,3- and 5-year survival rates of patients with low sEPO levels were $95.8,89$ and $89 \%$, respectively, while those of patients with high SEPO level were 84.2, 65.2 and 52.4\%, respectively. (D) In N0M0 patients there was no significant difference in DFS between those with low sEPO and those with high SEPO. EpoR, erythropoietin receptor; RCC, renal cell carcinoma; CSS, cause-specific survival; DFS, disease-free survival; sEPO, serum erythropoietin.

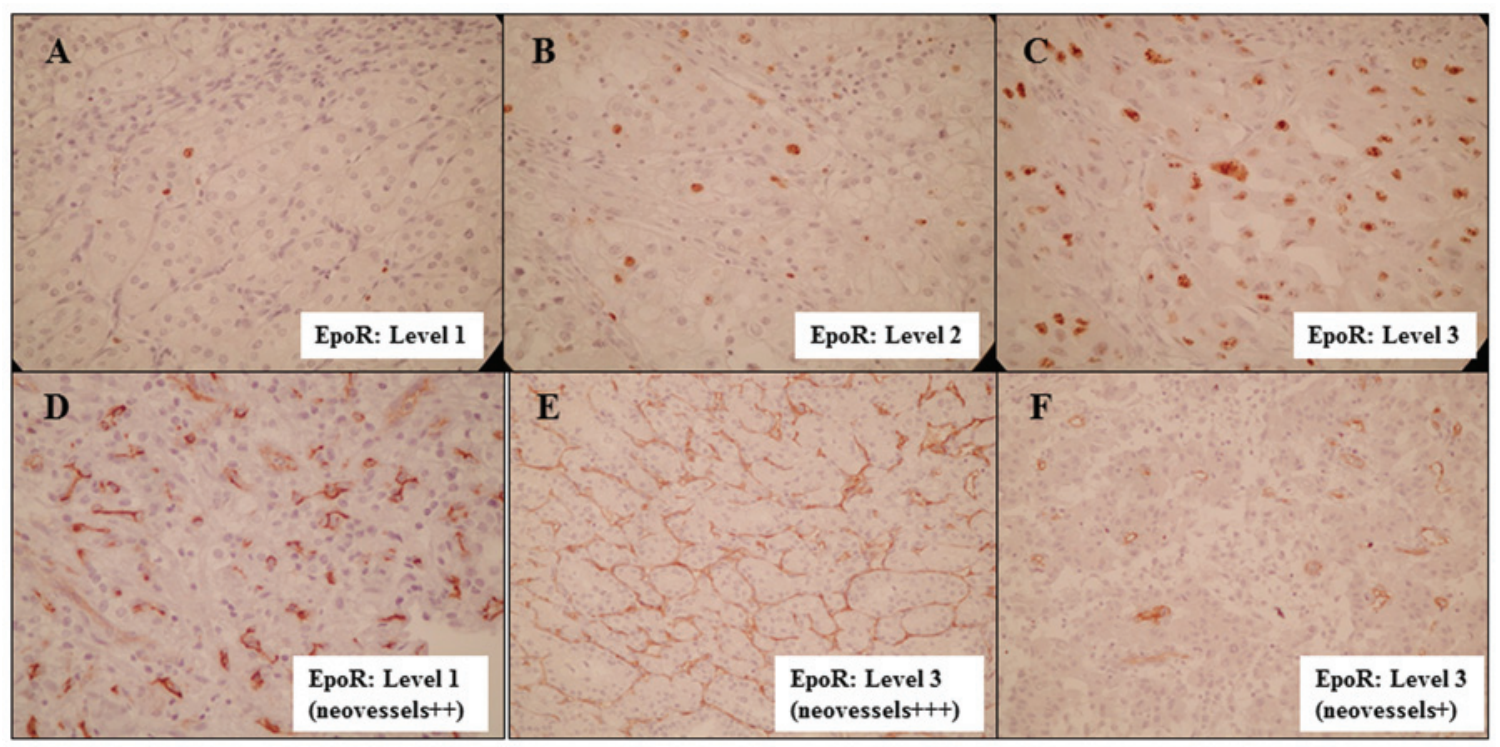

Figure 3. (A-C) Ki-67 immunostaining results used to evaluate proliferative status. The number of Ki-67-positive RCC cells per HPF was $7.7 \pm 1.0$ in level-1 tumors, $16.4 \pm 2.8$ in level-2 tumors ( $\mathrm{P}=0.0049$, compared to level 1) and 32.6 \pm 5.3 in level-3 tumors $(\mathrm{P}=0.0063$, compared to level 2). Tumors with higher EpoR expression levels had significantly larger numbers of Ki-67-positive cells. (D-F) CD34 immunostaining results used to evaluate angiogenesis. The number of CD34-positive neovessels per HPF was $22.1 \pm 2.5$ in level-1 tumors, $16.6 \pm 2.6$ in level-2 tumors and $19.2 \pm 3.5$ in level-3 tumors. (D) Various RCC specimens with low EpoR expression had a large number of neovessels and $(\mathrm{F})$ various specimens with high EpoR expression had a small number of neovessels. There was no correlation between the level of EpoR expression and the number of CD34-positive neovessels. Ki-67 staining and CD34 staining, reduced from x200. EpoR, erythropoietin receptor; RCC, renal cell carcinoma; HPF, high-power fields. 
Table II. Comparison of the clinocopathological factors between patients with low and high sEPO levels.

\begin{tabular}{|c|c|c|c|}
\hline & $\begin{array}{c}\mathrm{sEPO}<30 \mathrm{mU} / \mathrm{ml} \\
(\mathrm{n}=99)\end{array}$ & $\begin{array}{c}\mathrm{sEPO} \geq 30 \mathrm{mU} / \mathrm{ml} \\
(\mathrm{n}=39)\end{array}$ & $\mathrm{P}$-value \\
\hline Male/female & $72 / 27$ & $25 / 14$ & $0.3181^{\mathrm{a}}$ \\
\hline Age (years) & $60.5 \pm 11.4$ & $64.7 \pm 10.5$ & $0.0531^{\mathrm{b}}$ \\
\hline Side (right/left) & $44 / 55$ & $15 / 24$ & $0.5224^{\mathrm{a}}$ \\
\hline Size $(\mathrm{cm})$ & $5.1 \pm 3.1$ & $7.4 \pm 4.1$ & $0.0004^{\mathrm{b}}$ \\
\hline pT 1 or $2 / 3$ or 4 & $84 / 15$ & $21 / 18$ & $0.0001^{\mathrm{a}}$ \\
\hline $\mathrm{N}^{+}$ & $3(3.0 \%)$ & $6(15.4 \%)$ & $0.0081^{\mathrm{a}}$ \\
\hline $\mathrm{M}^{+}$ & $10(10.1 \%)$ & $11(28.2 \%)$ & $0.0081^{\mathrm{a}}$ \\
\hline Histological grade $3^{+}$ & $21(21.2 \%)$ & $20(51.3 \%)$ & $0.0005^{\mathrm{a}}$ \\
\hline $\mathrm{MVI}^{+}$ & $27(27.3 \%)$ & $28(71.8 \%)$ & $<0.0001^{\mathrm{a}}$ \\
\hline CRP (mg/dl) & $0.9 \pm 2.0$ & $3.6 \pm 5.6$ & $0.0010^{\mathrm{b}}$ \\
\hline
\end{tabular}

${ }^{a}$ Analyzed by the Chi-square test, banalyzed by the Mann-Whitney U test. sEPO, serum erythropoietin; pT, pathological T stage; $\mathrm{N}^{+}$, lymph node metastasis; $\mathrm{M}^{+}$, distant metastasis; MVI, microvascular invasion CRP, C-reactive protein.

A

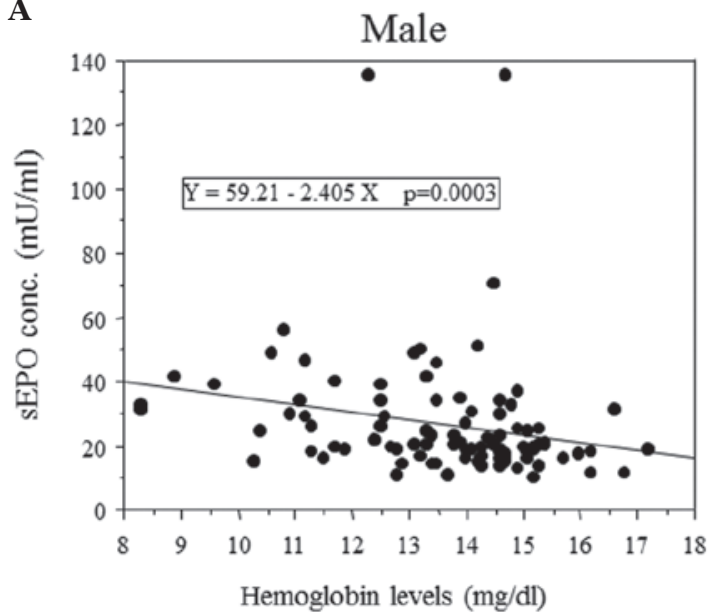

B

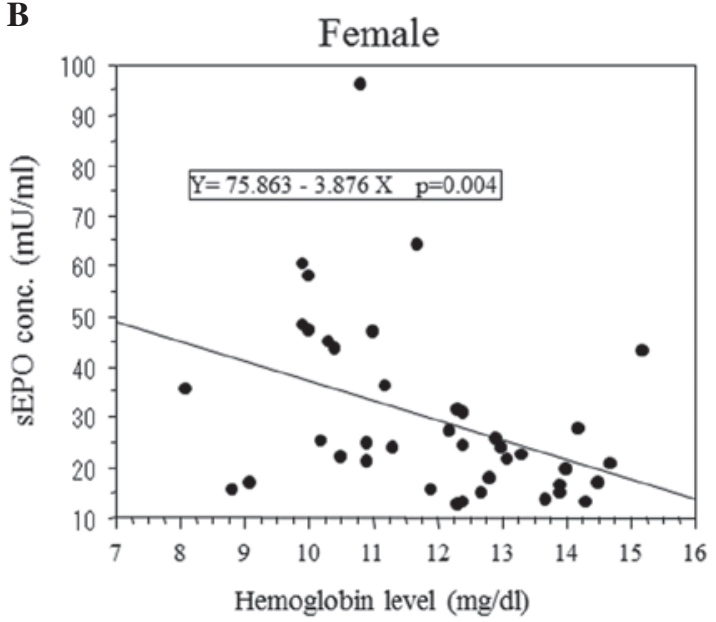

Figure 4. Correlation between sEPO levels and hemoglobin levels. EPO levels were inversely correlated with hemoglobin levels in both (A) male and (B) female patients. sEPO, serum erythropoietin; EPO, erythropoietin.

with high EpoR expression had a small number of neovessels (Fig. 3F). There was no correlation between levels of EpoR expression and the number of CD34-positive neovessels.

Association between EpoR expression levels and invasiveness. According to a previous study, invasiveness of RCC was evaluated in terms of pathological growth pattern; infiltrative or expansive (17). Seven of the 21 tumors with level-1 EpoR expression were infiltrative $(33 \%), 14$ of the 20 tumors with level-2 expression were infiltrative, and 10 of the 15 tumors with level-3 expression were infiltrative $(67 \%)(\mathrm{P}=0.0363$, Chi-square test). Infiltrative tumors contributed to a greater percentage of tumors with either level-2 or level-3 EpoR expression compared to tumors with level 1 EpoR expression. Therefore, increased EpoR expression appears to be associated with RCC invasiveness.

Comparison of clinicopathological factors and survival between patients with low and high sEPO levels. We also compared the clinicopathological factors of patients with low sEPO levels with those of patients with high sEPO levels (Table II). Patients with high sEPO had tumors with a significantly higher $\mathrm{pT}$ stage, size and histological grade, and also had higher CRP levels and higher percentages of metastatic disease and MVI $(\mathrm{P}<0.05)$. Patients with high sEPO had a significantly lower survival rate than those with low sEPO (Fig. 2C). The five-year survival rate for patients with low sEPO was $89 \%$, while for patients with high sEPO it was $52.4 \%$. In NOM0 patients $(n=110)$, however, DFS did not differ significantly between those with low SEPO and those with high sEPO (Fig. 2D).

Correlation between EpoR expression and sEPO levels. Of the 47 patients whose surgical specimens were evaluated for EpoR expression and whose sEPO levels were measured, the sEPO level was $17.3 \pm 6.4 \mathrm{mU} / \mathrm{ml}$ in patients whose tumors

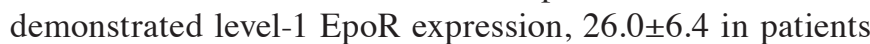
whose tumors demonstrated level-2 expression, and 36.2 \pm 7.7 
Table III. Logistic regression analysis for factors influencing sEPO elevation.

\begin{tabular}{|c|c|c|c|c|}
\hline \multirow[b]{2}{*}{ Variables } & \multirow{2}{*}{$\frac{\text { Univariate }}{\text { P-value }}$} & \multicolumn{3}{|c|}{ Multivariate } \\
\hline & & P-value & Odds ratio & Relative risk ratio $95 \% \mathrm{CI}$ \\
\hline Male & 0.3196 & & & \\
\hline Age & 0.052 & & & \\
\hline Tumor size & 0.0014 & 0.9150 & & \\
\hline Cell type & 0.5527 & & & \\
\hline (Clear cell vs. others) & & & & \\
\hline $\mathrm{pT} \geq 3$ & 0.0002 & 0.7951 & & \\
\hline Lymph node metastasis & 0.0166 & 0.7688 & & \\
\hline Distant metastasis & 0.0103 & 0.6958 & & \\
\hline Grade 3 component $^{+}$ & 0.0007 & 0.9201 & & \\
\hline $\mathrm{MVI}^{+}$ & $<0.0001$ & 0.0054 & 4.633 & $1.573-13.648$ \\
\hline Infiltrative type & 0.2234 & & & \\
\hline Anemia $^{+}$ & 0.0003 & 0.1425 & & \\
\hline $\mathrm{CRP} \geq 1 \mathrm{mg} / \mathrm{dl}$ & 0.0004 & 0.2601 & & \\
\hline
\end{tabular}

sEPO, serum erythropoietin; pT, pathological T stage; MVI, microvascular invasion; CRP, C-reactive protein.

A tumor size

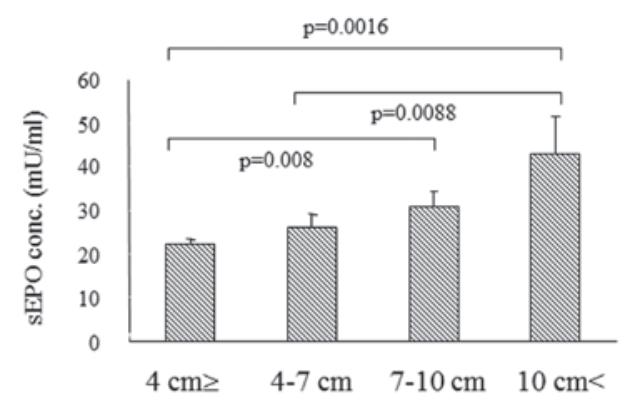

C distant metastasis

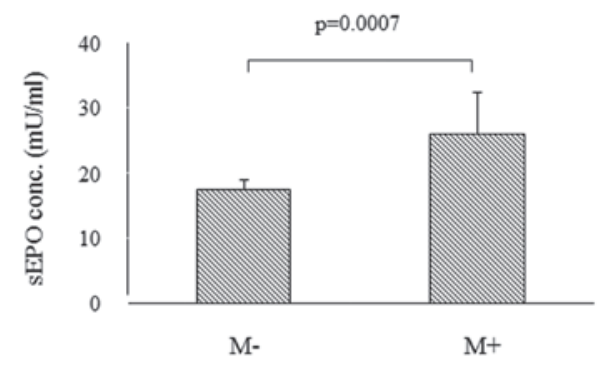

B pT stage

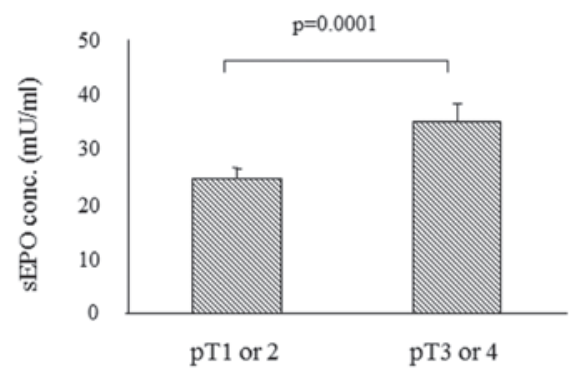

D TNM stage

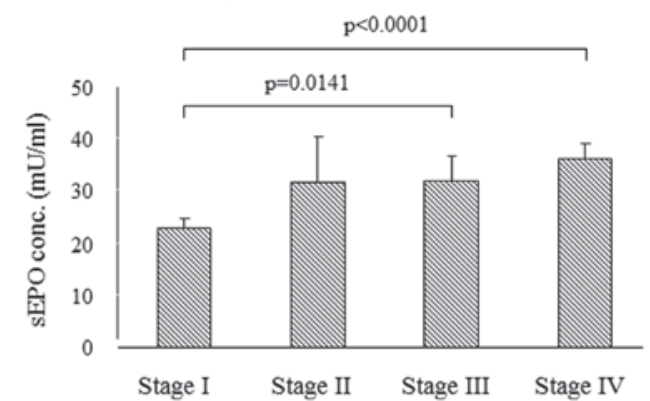

Figure 5. Correlation between sEPO level and clinicopathological factors. (A) Larger tumors, (B) higher pT stages, (C) distant metastases and (D) higher TNM stages were associated with higher levels of sEPO. EPO levels did not differ significantly between patients with either stage II, III or IV tumors. sEPO, serum erythropoetin; pT stage, pathological T stage.

in patients whose tumors demonstrated level-3 expression $(\mathrm{P}=0.0008$, compared to the level-1 value; $\mathrm{P}=0.053$, compared to the level-2 value). Serum EPO level increased in proportion to the increase in tissue EpoR expression level.

Negative correlation between sEPO levels and hemoglobin levels. As EPO stimulates erythrocyte production and the hemoglobin level is reportedly a prognostic factor in RCC patients, the correlation between sEPO level and hemoglobin level was evaluated. sEPO levels inversely correlated with hemoglobin levels in male and female patients (Fig. 4). The percentage of patients with anemia was greater in patients with high sEPO levels than those with low sEPO levels.

Factors associated with sEPO levels. To identify factors that influence sEPO level, we evaluated the correlation between sEPO level and various clinicopathological factors (Table III). As shown in Fig. 5, larger size, higher pT stage, distant 
A

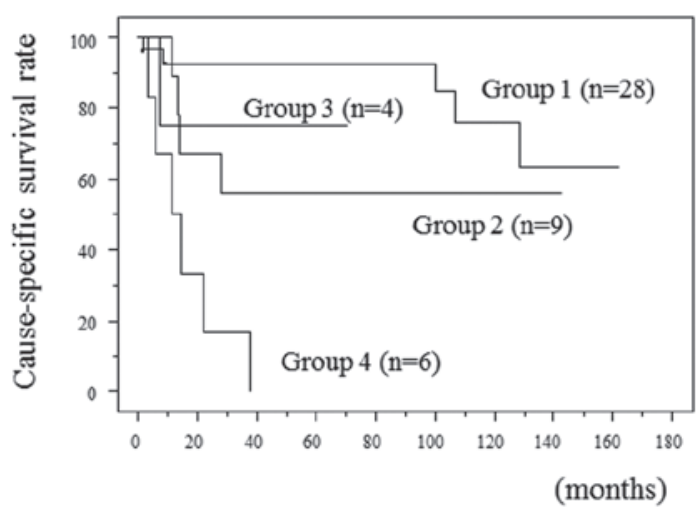

B

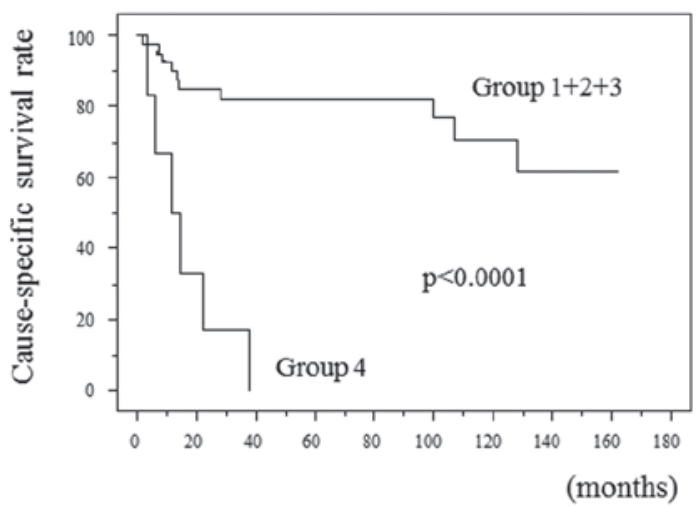

Figure 6. Kaplan-Meier curves for the four groups of 47 patients whose sEPO and specimen EpoR were determined. Group 1, low EpoR expression (level 1 or 2) and low sEPO (<30 mU/ml); group 2, high EpoR expression (level 3) and low sEPO; group 3, low EpoR expression and high sEPO ( $\geq 30 \mathrm{mU} / \mathrm{ml}$ ); group 4 , high EpoR expression and high sEPO. The prognosis for patients in group 4 was markedly poorer than that for patients in any of the other groups. The 1-, 2- and 3 -year survival rates for group-4 patients were 50, 33 and 17\%, respectively. SEPO, serum erythropoietin; EpoR, erythropoietin receptor.

metastasis (DM) and higher TNM stage were associated with sEPO elevation. Logistic regression analysis was used to identify independent factors associated with sEPO elevation. Univariate analysis demonstrated that tumor size, $\mathrm{pT} \geq 3$, lymph node metastasis (LNM), DM, presence of grade-3 component, MVI, anemia and $C R P \geq 1$ were significantly associated with sEPO elevation. In multivariate analysis, only MVI was independently associated with sEPO elevation. This result suggests that EPO-EpoR signaling might promote invasion of RCC cells into small venules or lymphatics. To confirm the association between MVI and sEPO elevation, we divided NOM0 patients with primary tumors $4-7 \mathrm{~cm}$ in size into 2 groups. Patients with MVI ( $\mathrm{n}=15$; mean tumor size, $5.5 \pm 0.2 \mathrm{~cm}$ ) had significantly higher sEPO levels than those without MVI $(\mathrm{n}=20$, mean tumor size, $5.4 \pm 0.2 \mathrm{~cm})(\mathrm{sEPO}$ level $=36.9 \pm 7.9 \mathrm{vs}$. $18.6 \pm 1.0 \mathrm{mU} / \mathrm{ml}$, respectively; $\mathrm{P}=0.0156)$. Although tumor size was similar in the two groups, patients with MVI had significantly higher sEPO levels than those without MVI.

Prognostic significance of increased EpoR expression and sEPO elevation in RCC patients. As described above, patients with tumors demonstrating higher EpoR expression appeared to have higher sEPO levels than those with lower EpoR expression. It is therefore possible that tumors with increased EpoR expression might have aggressive biological activities and have an increased ability to produce EPO. When patients with elevated sEPO have tumors with increased EpoR expression, the EPO-EpoR pathway might function effectively and increase the aggressiveness of the tumors. To examine this possibility, we divided the 47 patients whose sEPO and specimen EpoR were determined into 4 groups (Fig. 6). The patients with high EpoR expression and a high sEPO level had a worse prognosis than the other patients. Their 1-, 2- and 3-year CSS rates were only 50,33 and $17 \%$, respectively.

\section{Discussion}

In the current study, we firstly focused on EpoR expression in RCC tissue. Since increased EPO expression is associated with aggressiveness of various malignancies (3), increased EPO receptor expression might increase activity in the EPO-EpoR signaling pathway. EPO-EpoR coexpression in cancer tissue is reportedly an independent predictor for CSS in stage I non-small cell lung cancer (6). However, circulating EPO theoretically affects its receptor more than intracellular EPO does, and in a recent study intracellular (cytoplasmic) EPO expression was not correlated with the sEPO level (12). We therefore evaluated the sEPO level as well as tissue EpoR expression.

EPO-EpoR signaling reportedly stimulates proliferation in Caki-2 and 786-O RCC cells (2). In the present study, the degree of EpoR expression was correlated with the proliferative marker Ki-67. Several signal transduction pathways have been reported to be activated by the EPO-EpoR system. EPO-EpoR signaling activates the JAK-STAT pathway in head and neck squamous cell carcinomas (8), and Lester et al demonstrated that EPO activates the ERK/MAPK pathway in breast cancer cells (9). In the present study, the level of EpoR expression was correlated with proliferation, but not with the level of angiogenesis. Even RCC tissues with low EpoR expression frequently demonstrated prominent neovessels (Figs. 3D and F). Low-grade RCC is typically a highly vascular tumor, and in the present study, the sEPO level did not correlate with the number of CD34-positive cells $(\mathrm{P}=0.8078$, data not shown). This result is different from that obtained in a study of gastric cancer (18), in which EpoR level correlated with angiogenesis. RCC angiogenesis probably depends on other growth factors, including VEGF and PDGF.

RCC with high EpoR expression was found to frequently be infiltrative. This might be due to the fact that the EPO-EpoR pathway is associated with RCC invasion. In head and neck squamous cell carcinoma, JAK-STAT signaling contributes to cellular invasion (8). In our results, MVI was independently associated with elevated sEPO level but other factors, including tumor size, were not. In N0M0 patients with T1b tumor, patients with MVI had significantly higher sEPO levels than those without MVI despite similar tumor sizes between those 2 groups ( $36.9 \pm 7.9$ vs. $18.6 \pm 1.0 \mathrm{mU} / \mathrm{ml})$. Immunohistochemical analysis revealed that all 5 of the metastatic lesions examined demonstrated high EpoR expression (level 3) and that tumor cells in microvessels also demonstrated very high EpoR 
expression. EPO-EpoR signaling probably has a role in MVI, and thus in RCC metastasis, or RCC cells with high abilities of motility and invasion may produce larger amounts of EPO.

Renal tubular cells surrounding RCC demonstrated cytoplasmic EpoR expression, and EPO reportedly has a protective effect on renal tubular cells. In an ischemic acute injury model, the EPO system inhibited tubular cell apoptosis (19). The EPO-EpoR system is involved in the growth of cancer cells (2), the survival of stressed cancer cells, and probably in chemoresistance. EPO has also been reported to reduce cisplatin-induced apoptosis in RCC cells through a PKC-dependent pathway (20).

sEPO level and hemoglobin level were negatively correlated, but EPO usually stimulates the production of red blood cells. The presentation of polycythemia generally requires the presence of a high level of sEPO; a slightly elevated sEPO does not cause polycythemia. RCC patients with elevated sEPO levels tend to have advanced disease and thus may have elevated levels of various cytokines that cause anemia, including IL- 6 and TNF- $\alpha$. Ljungberg et al reported no association between SEPO level and erythrocytosis and reported a negative correlation between hemoglobin and sEPO level (4), but the RCC patients in their study were not divided into male and female groups despite differences in normal ranges of hemoglobin. In the present study, we found a negative correlation between sEPO and hemoglobin in both male and female patients.

As EPO and proteins IL-6, PDGF, VEGF and TGF- $\alpha$ are induced by HIF- $\alpha$, we evaluated the impact of EPO signaling on the prognosis of RCC patients, and found neither increased EpoR expression nor sEPO elevation to be an independent predictor for CSS. This is probably due to the fact that not only EPO, but various cytokines, were required for RCC progression. In the present study, we investigated the combination of tissue EpoR expression level and sEPO level. This combination can increase the accuracy with which the clinical outcome for RCC patients can be predicted. In patients with SEPO elevation and increased EpoR expression, EPO-EpoR signaling is possibly enhanced. Saintigny et al reported that EPO and EpoR coexpression was associated with poor survival in stage I non-small cell lung cancer (6). Although in multivariate analysis EPO-EpoR coelevation was not an independent factor for prognosis, the EPO-EpoR system appeared to be important in RCC progression. Further studies that include larger numbers of patients are required.

\section{References}

1. Da Silva JL, Lacombe C, Bruneval P, Casadevall N, Leporrier M, Camilleri JP, Bariety J, Tambourin P and Varet B: Tumor cells are the site of erythropoietin synthesis in human renal cancers associated with polycythemia. Blood 75: 577-582, 1990.
2. Westenfelder C and Baranowski RL: Erythropoietin stimulates proliferation of human renal carcinoma cells. Kidney Int 58: 647-657, 2000.

3. Hardee ME, Arcasoy MO, Blackwell KL, Kirkpatrick JP and Dewhirst MW: Erythropoietin biology in cancer. Clin Cancer Res 12: 332-339, 2006.

4. Ljungberg B, Rasmuson T and Grankvist K: Erythropoietin in renal cell carcinoma: evaluation of its usefulness as a tumor marker. Eur Urol 21: 160-163, 1992.

5. Michael A, Politi E, Havranek E, Corbishley C, Karapanagiotou L, Anderson C, Relph K, Syrigos KN and Pandha H: Prognostic significance of erythropoietin expression in human renal cell carcinoma. BJU Int 100: 291-294, 2007.

6. Saintigny P, Besse B, Callard P, Vergnaud AC, Czernichow S, Colombat M, Girard P, Validire P, Breau JL, Bernaudin JF and Soria JC: Erythropoietin and erythropoietin receptor coexpression is associated with poor survival in stage I non-small cell lung cancer. Clin Cancer Res 13: 4825-4831, 2007.

7. Acs G, Xu X, Chu C, Acs P and Verma A: Prognostic significance of erythropoietin expression in human endometrial carcinoma. Cancer 100: 2376-2386, 2004.

8. Lai SY, Childs EE, Xi S, Coppelli FM, Gooding WE, Wells A, Ferris RL and Grandis JR: Erythropoietin-mediated activation of JAK-STAT signaling contributes to cellular invasion in head and neck squamous cell carcinoma. Oncogene 24: 4442-4449, 2005.

9. Lester RD, Jo M, Campana WM and Gonias SL: Erythropoietin promotes MCF-7 breast cancer cell migration by an ERK/mitogen-activated protein kinase-dependent pathway and is primarily responsible for the increase in migration observed in hypoxia. J Biol Chem 280: 39273-39277, 2005.

10. Tilbrook PA, Colley SM, McCarthy DJ, Marais R and Klinken SP: Erythropoietin-stimulated Raf-1 tyrosine phosphorylation is associated with the tyrosine kinase Lyn in J2E erythroleukemic cells. Arch Biochem Biophys 396: 128-132, 2001.

11. Gong K, Zhang N, Zhang Z and Na Y: Coexpression of erythopoietin and erythopoietin receptor in sporadic clear cell renal cell carcinoma. Cancer Biol Ther 5: 582-585, 2006.

12. Papworth K, Bergh A, Grankvist K, Ljungberg B and Rasmuson T: Expression of erythropoietin and its receptor in human renal cell carcinoma. Tumour Biol 30: 86-92, 2009.

13. Ito K, Yoshii H, Asakuma J, Sato A, Horiguchi A, Sumitomo M, Hayakawa M and Asano T: Clinical impact of the presence of the worst nucleolar grade in renal cell carcinoma specimens. Jpn J Clin Oncol 39: 588-594, 2009.

14. Horiguchi A, Asano T, Asakuma J, Asano T, Sumitomo M and Hayakawa M: Impact of caveolin-1 expression on clinicopathological parameters in renal cell carcinoma. J Urol 172: 718-722, 2004.

15. Sturiale A, Campo S, Crascì E, Coppolino G, Bolignano D, Grasso G and Buemi M: Erythropoietin and its lost receptor. Nephrol Dial Transplant 22: 1484-1485, 2007.

16. Miyajima A, Kosaka T, Asano T, Asano T, Seta K, Kawai T and Hayakawa M: Angiotensin II type I antagonist prevents pulmonary metastasis of murine renal cancer by inhibiting tumor angiogenesis. Cancer Res 62: 4176-4179, 2002.

17. Islam AH, Ehara T, Kato H, Hayama M, Kobayashi S, Igawa Y and Nishizawa O: Calponin h1 expression in renal tumor vessels: correlations with multiple pathological factors of renal cell carcinoma. J Urol 171: 1319-1323, 2004.

18. Ribatti D, Marzullo A, Nico B, Crivellato E, Ria R and Vacca A: Erythropoietin as an angiogenic factor in gastric carcinoma. Histopathology 42: 246-250, 2003.

19. Vesey DA, Cheung C, Pat B, Endre Z, Gobé G and Johnson DW: Erythropoietin protects against ischaemic acute renal injury. Nephrol Dial Transplant 19: 348-355, 2004.

20. Li J, Vesey DA, Johnson DW and Gobe G: Erythropoietin reduces cisplatin-induced apoptosis in renal carcinoma cells via a PKC dependent pathway. Cancer Biol Ther 6: 1944-1950, 2007. 